\title{
Reparo Transperineal de Retocele - Avaliação do Grau de Satisfação, Dispareunia e Recidiva Pós-Operatória
}

\author{
Transperineal Repair for Rectocele - Grade of Satisfaction and \\ Postoperatory Dispareunia and Recurrence
}

\author{
SINARA MÔNICA DE OLIVEIRA LEITE ${ }^{1}$, RODRIGO GUIMARÃES OLIVEIRA ${ }^{2}$, FLÁVIA FONTES FARIA ${ }^{2}$, \\ ANTÔNIOCARLOS BARROS LIMA JÚNIOR ${ }^{2}$, FÁBIO GONTIJO RODRIGUES'2, \\ ÁUREACÁSSIA GUALBERTO BRAGA ${ }^{1}$, GERALDO MAGELAGOMES DACRUZ ${ }^{3}$ \\ ${ }^{1}$ Assistentes, ${ }^{2}$ Residentes e especializandos, ${ }^{3}$ Coordenador do Grupo de Coloproctologia da Santa Casa \\ de Belo Horizonte e Faculdade de Ciências Médicas de Minas Gerais.
}

\begin{abstract}
LEITE SMO; OLIVEIRA RG; FARIA FF; LIMA JÚNIOR ACB; RODRIGUES FG; BRAGA ACG; CRUZ GMG. Reparo Transperineal de Retocele - Avaliação do Grau de Satisfação, Dispareunia e Recidiva Pós-Operatória. Rev bras Coloproct, 2009;29(4): 458-465.

RESUMO: A retocele é uma disfunção pélvica pobremente diagnosticada, apesar de sua prevalência significativa. Seu reconhecimento é essencial para o tratamento de determinados casos, como constipação refratária. A sintomatologia é vaga, e nem sempre associada ao prolapso. O tratamento clínico é ineficaz quando utilizado isoladamente. Existem várias técnicas, e dentre elas a abordagem transperineal pode ser considerada uma opção adequada na correção do prolapso. Nas 12 pacientes objetos de nosso estudo, houve melhora significativa do padrão evacuatório, uma resposta aceitável no tratamento da dispareunia, com índice de recidiva tolerável e alto grau de satisfação pós-operatória. A comparação com as demais técnicas ainda exige estudos comparativos mais significativos, com amostras mais expressivas. Até o momento, qualquer análise comparativa entre as técnicas cirúrgicas utilizadas pode ser falha.
\end{abstract}

Descritores: Reparo transperineal; retocele; grau de satisfação; dispareunia; recidiva.

\section{INTRODUÇÃO}

Prolapsos de assoalho pélvico (PAP) são responsáveis por \pm 300.000 cirurgias anuais nos Estados Unidos, gerando um custo de \pm 1 bilhão de dólares ao ano. A mulher apresenta um risco de $11,1 \%$, durante sua vida, de desenvolver desordem de assoalho pélvico. Além disso, estima-se que os PAP terão a incidência aumentada em $45 \%$ em um futuro próximo, sendo considerado, em breve, como principal motivo de consulta relacionadas aos distúrbios do assoalho pélvico.

A retocele é uma desordem ainda pouco compreendida, e ainda não há procedimentos padronizados para diagnóstico. A literatura mundial mostra grande variação na incidência dos PAP, entre 30 e $93 \%$ das mulheres, variando conforme idade, paridade e tipo de parto realizado. Além disso, acrescenta-se o fato de que poucos PAP são sintomáticos.
A abordagem cirúrgica do PAP é uma opção de tratamento, porém, sabe-se que a resolução da falha anatômica da musculatura pélvica não necessariamente elimina os sintomas previamente associados. A recidiva após cirurgia para abordagem do PAP é elevada, admitindo Olsen et al. (1997) uma taxa de recorrência pós-operatória de $\pm 29,9 \%$, sendo uma das causas dessa alta incidência a grande variedade de técnicas cirúrgicas utilizadas no reparo de retoceles. Há poucos trabalhos comparativos (geralmente retrospectivos e com pequena amostra populacional) entre as técnicas cirúrgicas empregadas, e uma indefinição sobre a indicação de determinada técnica para cada tipo específico de paciente e de morbidade.

O estudo ora proposto objetiva avaliar o grau de satisfação de pacientes submetidas ao "reparo cirúrgico transperineal de retoceles", assim como avaliar dispareunia pós-operatória e recidiva de sintomas.

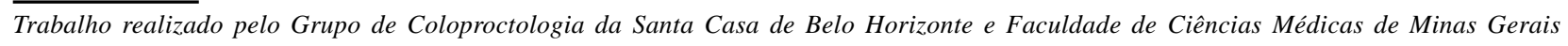


Rev bras Coloproct

Outubro/Dezembro, 2009
Reparo Transperineal de Retocele - Avaliação do Grau de Satisfação, Dispareunia e Recidiva Pós-Operatória Sinara Mônica de Oliveira Leite e Cols.
Vol. 29

\section{OBJETIVOS}

Realizar um estudo retrospectivo visando avaliar o grau de satisfação, resolução de sintomas, presença de dispareunia e recidiva pós operatória após reparo transperineal de retocele (RTPR).

\section{MÉTODO}

Foram estudados 16 pacientes submetidas ao RTPR no período de 1997 a 2008 (tabela 2). As pacientes foram contatadas por telefone, sendo informadas da realização da pesquisa e de seus objetivos, assim como obtenção verbal de consentimento informado. Doze pacientes responderam verbalmente a um questionário padrão, cujas variáveis analisadas foram:

1. Dados gerais (idade, estado civil e raça);

2. Número de partos;

3. Fase do climatério na qual se encontram;

4. Hábito intestinal pré e pós-reparo cirúrgico;

5. Necessidade de auxílio manual durante a evacuação;

6. Presença de doenças anais associadas;

7. Histórico de cirurgias prévias;

8. Tempo decorrido desde a abordagem cirúrgica;

9. Atividade sexual;

10. Presença ou não de dispareunia;

11. Grau de satisfação com o reparo cirúrgico;

12. Recidiva dos sintomas.

\section{RESULTADOS}

Das 16 pacientes estudadas conseguiu-se contato telefônico com 12, que concordaram em responder verbalmente ao questionário proposto.

Idade: a idade média foi de 57,8 anos, variando de 41 a 88 anos. As pacientes entre 51 e 70 anos representaram $66,7 \%$ dos casos. Nenhuma paciente abordada cirurgicamente tinha menos de 40 anos de idade (tabela 1).

Raça: $42 \%$ das pacientes eram de da raça branca, respondendo pacientes de cor parda por $58 \%$ (tabela 3).

Partos: a média de gestação foi de três por paciente, variando de 0 a 11 gestações. Quanto ao tipo de parto realizado, nota-se uma maior prevalência de
Tabela 1 - Distribuição das pacientes submetidas à cirurgia para abordagem de retocele entre 1997 e 2008 segundo a idade.

\begin{tabular}{lccc}
\hline Idade (anos) & N & \% & Média (anos) \\
\hline$<20$ & 0 & 0 & \\
$21-30$ & 0 & 0 & \\
$31-40$ & 0 & 0 & \\
$41-50$ & 3 & 25 & 57,83 \\
$51-60$ & 4 & 33,33 & \\
$61-70$ & 4 & 33,33 & \\
$71-80$ & 0 & 0 & \\
$>81$ & 1 & 8,34 & \\
\hline
\end{tabular}

Tabela 2 - Distribuição das pacientes submetidas à cirurgia para abordagem de retocele entre 1997 e 2008 segundo o estado civil.

\begin{tabular}{lcc}
\hline Estado civil & N & \% \\
\hline Solteiras & 0 & 0 \\
Casadas & 9 & 75 \\
Viúvas & 2 & 17 \\
Divorciadas & 1 & 8 \\
\hline
\end{tabular}

Tabela 3 - Distribuição das pacientes submetidas à cirurgia para abordagem de retocele entre 1997 e 2008 segundo a raça.

\begin{tabular}{lcc}
\hline Raça & N & \% \\
\hline Negra & 0 & 0 \\
Branca & 5 & 42 \\
Parda & 7 & 58 \\
\hline
\end{tabular}

partos normais em relação às cesarianas (24 partos normais e 11 cesarianas). Oito das 12 pacientes estudadas foram submetidas a partos pelas duas técnicas, e apenas quatro pacientes foram submetidas a apenas partos naturais (gráfico 1).

Menopausa: $50 \%$ das pacientes já estavam em climatério (tabela 4).

Hábito intestinal no pré-operatório: $O$ hábito intestinal pré-operatório das pacientes foi avaliado quanto à frequência evacuatória e consistência fecal. Das pacientes avaliadas sete (58,3\% dos casos) apre- 


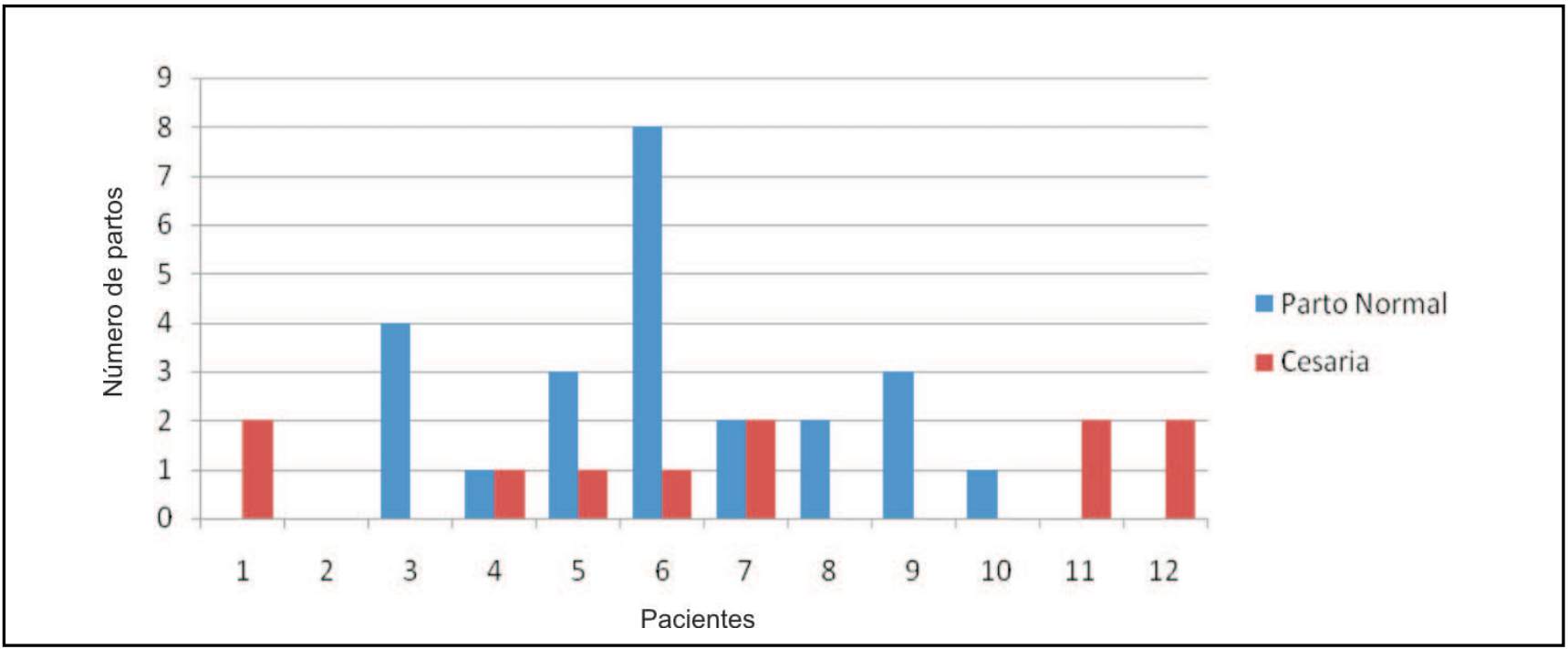

Gráfico 1 - Distribuição das pacientes submetidas à cirurgia para abordagem de retocele entre 1997 e 2008 segundo as modalidades de partos a que foram submetidas.

Tabela 4 - Distribuição das pacientes submetidas à cirurgia para abordagem de retocele entre 1997 e 2008 segundo a o período de menopausa.

\begin{tabular}{lll}
\hline Menopausa & N & \% \\
\hline Antes & 3 & 25 \\
Climatério & 3 & 25 \\
Depois & 6 & 50 \\
\hline
\end{tabular}

sentavam intervalo evacuatório maior que 3 dias, variando de 4 a 15 dias. $75 \%$ das pacientes apresentavam fezes ressecadas (gráfico 2, tabela 5). A necessidade de auxílio manual intravaginal durante $\mathrm{o}$ ato evacuatório ocorreu em 91,7\% dos casos (tabela 6).

Doenças anais associadas: Apenas uma paciente $(8,3 \%)$ apresentava doença anal associada, tratando-se de doença hemorroidária de primeiro grau.

Cirurgias prévias: seis pacientes $(50 \%)$ das pacientes não apresentavam histórico de cirurgias anais; 25\% admitiram cirurgias anais prévias; 4 paciente admitiram histerectomia $(33,3 \%), 2$ admitiram perineoplastia $(16,67 \%)$ e uma paciente correção de cistocele $(8,3 \%)$ (tabela 7$)$.

Pós-operatório: O tempo de pós-operatório variou de três meses a nove anos, com média de 3,75 anos. Aproximadamente $42 \%$ das pacientes apresentavam período pós-operatório menor que um ano (tabela 8$)$.

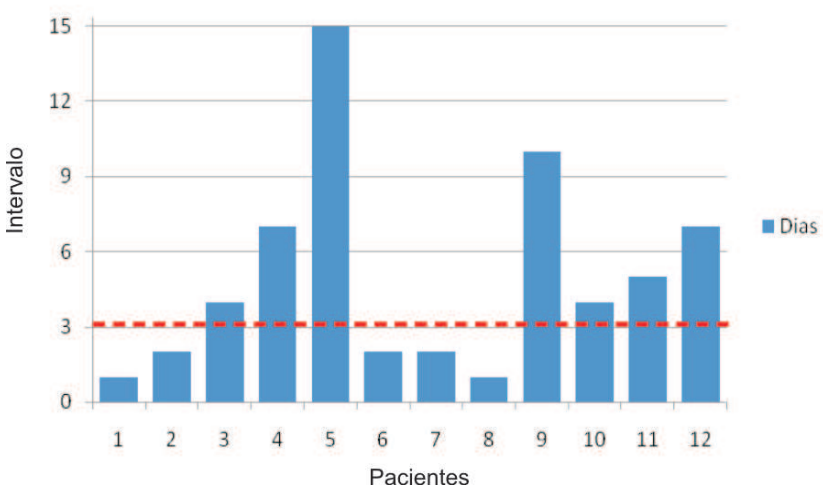

Gráfico 2 - Distribuição das pacientes submetidas à cirurgia para abordagem de retocele entre 1997 e 2008 segundo a frequência evacuatória em dias, avaliada no pré-operatório.

Tabela 5 - Distribuição das pacientes submetidas à cirurgia para abordagem de retocele entre 1997 e 2008 segundo a consistência fecal no pré-operatório.

\begin{tabular}{lcc}
\hline Consistência fecal & N & \% \\
\hline Ressecadas & 9 & 75 \\
Pastosas & 3 & 25 \\
Líquidas & 0 & 0 \\
\hline
\end{tabular}

Hábito intestinal no pós-operatório: o hábito intestinal foi reavaliado no pós-operatório, verificando-se que o intervalo evacuatório foi normalizado 
Rev bras Coloproct

Outubro/Dezembro, 2009
Reparo Transperineal de Retocele - Avaliação do Grau de Satisfação, Dispareunia e Recidiva Pós-Operatória Sinara Mônica de Oliveira Leite e Cols.
Vol. 29 $\mathbf{N}^{\circ} 4$
Tabela 6 - Distribuição das pacientes submetidas à cirurgia para abordagem de retocele entre 1997 e 2008 segundo a necessidade de ajuda manual intravaginal durante o ato evacuatório no pré-operatório.

\begin{tabular}{lrc}
\hline Necessidade de ajuda manual & $\mathbf{N}$ & $\boldsymbol{\%}$ \\
\hline Sim & 11 & 91,67 \\
Não & 1 & 8,33 \\
\hline
\end{tabular}

Tabela 7 - Distribuição das pacientes submetidas à cirurgia para abordagem de retocele entre 1997 e 2008 segundo ocorrências de cirurgias prévias.

\begin{tabular}{lcl}
\hline Cirurgias associadas & N & \\
\hline Correção de cistocele & 1 & $(8,33 \%)$ \\
Perineoplastia & 2 & $(16,67 \%)$ \\
Histerectomia & 4 & $(33,33 \%)$ \\
Cirurgias anais & 3 & $(25 \%)$ \\
Nenhuma & 6 & $(50 \%)$ \\
\hline
\end{tabular}

Tabela 8 - Distribuição das pacientes submetidas à cirurgia para abordagem de retocele entre $1997 e$ 2008 segundo o tempo de pós-operatório após a correção de retocele.

\begin{tabular}{llcc}
\hline $\begin{array}{l}\text { Tempo de pós- } \\
\text { operatório (anos) }\end{array}$ & N & $\%$ & Média (anos) \\
\hline$<1$ & 5 & 41,68 & \\
2 & 1 & 8,33 & \\
3 & 0 & 0 & \\
4 & 2 & 16,67 & \\
5 & 0 & 0 & 3,75 \\
6 & 1 & 8,33 & \\
7 & 1 & 8,33 & \\
8 & 1 & 8,33 & \\
9 & 1 & 8,33 & \\
\hline
\end{tabular}

em todos os casos, sendo que nenhuma paciente apresentava intervalo evacuatório maior que 3 dias no pósoperatório, e em $75 \%$ dos casos a evacuação era diária. Todas as pacientes apresentavam fezes de consistência pastosa (gráfico 3, tabela 09) e nenhuma paciente necessitava de ajuda manual intravaginal durante as evacuações.

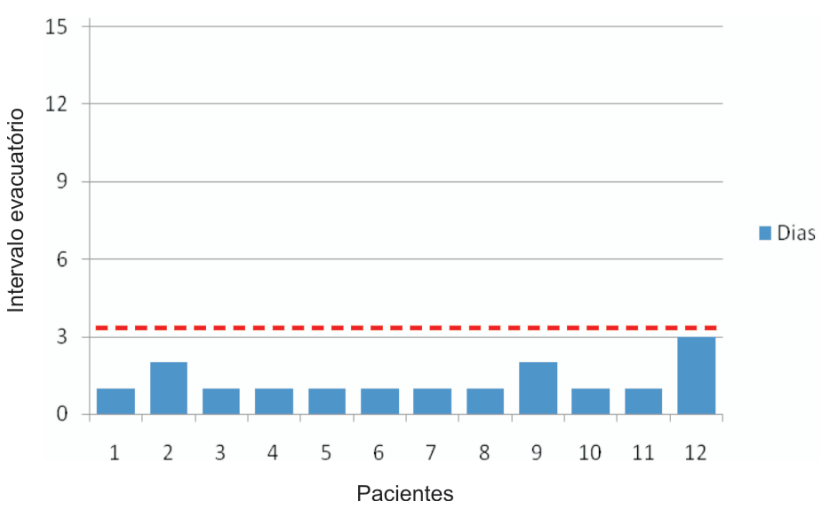

Gráfico 3 - Distribuição das pacientes submetidas à cirurgia para abordagem de retocele entre 1997 e 2008 segundo a frequência evacuatória em dias, avaliada no pós-operatório.

Tabela 9 - Distribuição das pacientes submetidas à cirurgia para abordagem de retocele entre 1997 e 2008 segundo a consistência fecal no pós-operatório.

\begin{tabular}{lrr}
\hline Consistência fecal & N & \% \\
\hline Ressecadas & 0 & 0 \\
Pastosas & 12 & 100 \\
Líquidas & 0 & 0 \\
\hline
\end{tabular}

Prolapso mucoso no pós-operatório: nenhuma paciente apresentou prolapso mucoso após correção cirúrgica da retocele. Apenas uma paciente (11\%) desenvolveu doença hemorroidária após a abordagem cirúrgica.

Vida sexual e dispareunia: nove pacientes (75\%) ainda apresentam vida sexual ativa, e destas dois terços não se queixavam de dispareunia, mesmo na presença de retocele. Das três pacientes que se queixaram de dispareunia duas relataram tal sintoma antes da correção cirúrgica e uma antes e mesmo após a correção da retocele. $O$ grau da dor foi classificada através de uma escala de notas numerada de 1 a 5 , sendo a nota 1 para dor leve e nota 5 para dor insuportável. Das três pacientes que apresentaram dispareunia duas queixaram-se de dor grau 2 e uma de dor grau 5 .

Recidiva dos sintomas: não houve recidiva dos sintomas em $75 \%$ das pacientes. Os três casos de recidivas ocorreram após seis meses, dois anos e três anos, respectivamente, após a correção cirúrgica da retocele.

Grau de satisfação: todas as pacientes $(100 \%)$ afirmaram um grau de satisfação adequado para a cirurgia a que foram submetidas (tabela 10). 
Rev bras Coloproct Outubro/Dezembro, 2009
Reparo Transperineal de Retocele - Avaliação do Grau de Satisfação, Dispareunia e Recidiva Pós-Operatória

Sinara Mônica de Oliveira Leite e Cols.
Vol. 29

\section{DISCUSSÃO}

A retocele consiste na herniação da parede anterior do reto através da parede posterior da vagina, de incidência controversa. Estudos demonstram que 30 a 93\% das mulheres avaliadas em caráter ambulatorial apresentam certo grau de prolapso de órgãos pélvicos ${ }^{11}$. Entretanto, esta estatística não é a vivenciada no cotidiano, sendo altamente subestimada, já que são diagnosticados apenas os casos sintomáticos. Além do mais, na maioria dos casos detectados, o prolapso é pequeno, não alcançando o intróito vaginal. Os casos de retocele grau III (aqueles que ultrapassam o intróito vaginal) são evidenciados em apenas 5\% dos $\operatorname{casos}^{10}$. Entretanto, dentre as pacientes que procuram auxílio médico com queixas de distúrbios do assoalho pélvico, 20 a $80 \%$ das mesmas apresentam algum grau de retocele, que pode ser ou não a causa dos sintomas ${ }^{1}$. A retocele é encontrada em $15 \%$ das pacientes com constipação refratária ao tratamento clínico ${ }^{1}$.

A patogenia também ainda é discutida. Muito se avalia sobre a existência anatômica do septo retovaginal, também denominado fáscia endopélvica ou fáscia de Denonvillier. Trata-se de uma estrutura oriunda da fusão das duas paredes do fundo de saco peritonial embriológico, composta de tecido fibromuscular que recobre a parede posterior da vagina e se fixa em estruturas adjacentes, oferecendo sustentação aos órgãos pélvicos e exercendo função de barreira contra o prolapso. Uma das hipóteses para a patogênese da retocele seria uma falha na fáscia retovaginal, fato comprovado por Richardson em estudos realizados em cadáveres ${ }^{12}$.

No presente estudo, foram analisadas 12 pacientes submetidas a reparo transperineal através de plástica dos músculos elevadores do ânus, sem uso de prótese. O grupo alocado apresentava faixa etária variando de 41 a 88 anos, com média de 57,3 anos. A maioria das mulheres era casada e da raça parda.

Dos 12 casos estudados, apenas uma era nulípara. Aproximadamente dois terços das pacientes multíparas foram submetidas a no mínimo 1 parto vaginal, com média de 2 partos normais por paciente. Tal achado favorece a hipótese de que a multiparidade, principalmente quando associada ao parto vaginal, predispõe ao surgimento de retoceles devido a estiramento das estruturas pélvicas na passagem do neonato pelo canal vaginal, podendo cursar com lacerações da fáscia retovaginal.
Tabela 10 - Distribuição das pacientes submetidas à cirurgia para abordagem de retocele entre 1997 e 2008 segundo o grau de satisfação com a correção cirúrgica de retocele.

\begin{tabular}{lcc}
\hline Grau de satisfação & $\mathbf{N}$ & $\mathbf{\%}$ \\
\hline Muito satisfeita & 8 & 66,67 \\
Satisfeita & 4 & 33,33 \\
Razoável & 0 & 0 \\
Insatisfeita & 0 & 0 \\
\hline
\end{tabular}

Associado a multiparidade, nota-se a maior prevalência dos casos (50\% das pacientes estudadas) em mulheres menopausadas. $\mathrm{O}$ envelhecimento e a disfunção hormonal estão associados a uma menor produção de colágeno, além de uma menor elasticidade dos tecidos frouxos. Assim, existe maior risco de lacerações da fáscia retovaginal e menor sustentabilidade dos órgãos pelo assoalho pélvico, predispondo ao prolapso.

A sintomatologia é vaga e, quando presente, é importante o diagnóstico diferencial. A presença de retocele não necessariamente significa que esta seja a causa dos sintomas de disfunção pélvica. Retoceles avançadas estão associadas a múltiplos e inespecíficos sintomas, incluindo dificuldade e esforço evacuatório, constipação, massa vaginal durante a evacuação, dor e sangramento retal, sensação de evacuação incompleta, mucorréia, soiling e dispareunia.

Estudos referem que 75 a $100 \%$ das pacientes portadoras de retocele são constipadas crônicas, o que é caracterizado por intervalo evacuatório maior que 3 dias e fezes de consistência ressecada ${ }^{1}$. A estatística obtida com este estudo refere que $58,33 \%$ das pacientes apresentam intervalo evacuatório maior que 3 dias (variando de 4 a 15 dias), e $75 \%$ das mesmas tinham fezes de consistência ressecada.

A necessidade de auxílio manual intravaginal durante a defecação é um achado comum, ocorrendo em 20 a $75 \%$ dos casos $^{1}$. Em estudo publicado por Fisher et al, a queixa de digitação ocorreu em um terço dos casos avaliados ${ }^{2}$. É a sintomatologia mais importante, e a que define a indicação do tratamento cirúrgico da retocele. Neste estudo, notou-se uma incidência elevada deste sintoma, ocorrendo em $91,67 \%$ das pacientes.

O histórico de cirurgias pélvicas e perineais tem grande influência na patogenia e nos resultados da cor- 
reção cirúrgica de retocele. A necessidade de cirurgias prévias no assoalho pélvico, tais como perineoplastia e cirurgias anais, já sugere um enfraquecimento das estruturas de sustentação, elevando a probabilidade de desenvolvimento de retocele. Cirurgias pélvicas, tais como histerectomia e correção de cistocele envolvem dissecção pélvica, com lesão de estruturas de sustentação por vezes já atrofiadas, predispondo também ao surgimento de prolapsos. Das pacientes analisadas, $62,5 \%$ já haviam sido submetidas a alguma cirurgia pélvica ou perineal, dentre elas histerectomia, correção de cistocele, cirurgias anais e perineoplastia.

O resultado funcional do tratamento de retocele é altamente dependente da seleção dos pacientes. Deve-se abordar apenas os casos de retoceles sintomáticas. O uso de procinéticos e dieta de alto resíduo é descrito na literatura e pode amenizar os sintomas obstrutivos ${ }^{1,2}$. Porém, o tratamento clínico é pouco eficaz quando utilizado isoladamente.

Várias técnicas cirúrgicas são descritas, todas evidenciando resultados satisfatórios. Não existe uma única técnica que se sobreponha às demais. Estudos demonstram que ginecologistas têm preferência pela abordagem transvaginal, porém esta apresenta maior incidência de dispareunia pós-operatória ( $25 \%$ dos casos), freqüente necessidade de reoperação e baixa eficácia na resolução da disfunção evacuatória ${ }^{1,2,3,4}$. Coloproctologistas geralmente utilizam o reparo transanal, seja através de sutura obliterativa (técnica de Block), reparo transretal com plicatura da camada muscular do reto (procedimento de Sarles) ou plicatura grampeada da mucosa retal redundante (STARR procedure) $)^{1,2,3,4}$. Entretanto, devido a grande manipulação anal envolvendo dilatação esfincteriana, existe um maior índice de lesões e maior risco de incontinência anal pós-operatória, além de não permitir esfincteroplastias associadas, estando contraindicada em pacientes com incontinência fecal. Para esses casos, geralmente se reserva a técnica por acesso transperineal. Estudos demonstram que não há benefício adicional com o uso de próteses no que diz respeito aos resultados pós-operatórios ${ }^{8,13}$.

A técnica utilizada no presente estudo foi o reparo transperineal com plástica da musculatura elevadora do ânus e dos músculos perineais. Consiste em incisão transversal perineal, com dissecção através do septo retovaginal até a cúpula vaginal, próximo ao colo uterino, identificando a falha entre os músculos elevadores do ânus. Posteriormente, realiza-se a plicatura dos elevadores com fio de absorção lenta (poliglactina 1) até o nível do anel anorretal, fechamento da musculatura perineal e da pele com pontos separados. Eventualmente, resseca-se a mucosa vaginal redundante.

O tempo de pós-operatório das pacientes avaliadas foi de, em média, 3,75 anos, variando de 3 meses a 9 anos. A maioria dos estudos apresenta curto período de acompanhamento pós-operatório ${ }^{2,3,5}$, geralmente menor que 3 a 4 anos.

Na reavaliação pós-operatória do hábito intestinal, o intervalo evacuatório de todas as pacientes foi menor que 3 dias, associadas a fezes pastosas. Associado a isso, a necessidade de digitação intravaginal durante o ato evacuatório foi resolvido em $100 \%$ dos casos. Em trabalho publicado por Fischer ${ }^{2,3}$, a técnica que apresenta a melhor resposta na correção de distúrbios evacuatórios é a abordagem transperineal; $87,5 \%$ dos casos apresentaram melhora do padrão evacuatório na abordagem transperineal, enquanto que $57,14 \%$ na abordagem transvaginal, e $44,44 \%$ na transretal. A digitação transvaginal, avaliada no mesmo trabalho, foi resolvida em apenas $62,5 \%$ dos casos após abordagem transvaginal, e em $25 \%$ dos casos na abordagem transretal. Em revisão publicada por $\mathrm{Kahn}^{4}$, a melhora da disfunção evacuatória com a abordagem transretal variou entre 46 e $84 \%$ dos casos. Após reparo transperineal com uso de prótese, Mercer-Jones ${ }^{5}$ constatou que $31,81 \%$ das pacientes ainda apresentavam constipação, e que $45,45 \%$ das mesmas ainda necessitavam de digitação intravaginal para auxílio na defecação.

Nenhuma paciente deste estudo apresentou prolapso mucoso, seja anal ou vaginal. Como já relatado na técnica, raros foram os casos nos quais foi necessária ressecção vaginal. Em nenhum caso foi realizada ressecção de mucosa retal redundante. Assim, de acordo com estes dados, pode-se supor que a ressecção de mucosa retal redundante não é benéfica, além de trazer riscos como deiscência de sutura e septicemia, como já relatado na abordagem transretal ${ }^{2,3}$.

Dentre as pacientes sexualmente ativas, aproximadamente $33 \%$ apresentavam queixa de dispareunia. Após a correção cirúrgica transperineal, apenas 1 (um terço dos casos sintomáticos) manteve o sintoma no pós-operatório, classificando a dor como leve; em nenhum caso foi constatada dispareunia em pacientes previamente assintomáticas. Em revisão publicada por Geoffrey $^{7}$, após abordagem transvaginal, a dispareunia 
Rev bras Coloproct Outubro/Dezembro, 2009
Reparo Transperineal de Retocele - Avaliação do Grau de Satisfação, Dispareunia e Recidiva Pós-Operatória Sinara Mônica de Oliveira Leite e Cols. se manteve entre 25 e 68,66\% dos casos; dentre os casos resolvidos, houve recidiva do sintoma de 0 a $7 \%$. $\mathrm{Na}$ abordagem transretal, os resultados foram frustrantes. A freqüência da dispareunia aumentou em 2 trabalhos analisados, com incidência de 9,8 a 49,44\%. Sehapayak ${ }^{14}$ constatou que $9,8 \%$ das pacientes previamente sem dispareunia desenvolveram o mesmo após correção transretal da retocele. Boccasanta ${ }^{8}$ também demonstra piora da dispareunia após correção transretal da retocele.

Não foram constatadas complicações pós-operatórias no presente estudo.

O grau de satisfação obtido com este estudo foi adequado, já que $100 \%$ das pacientes relataram-se satisfeitas com o resultado após abordagem transperineal. Tal fato demonstra que, mesmo com a queixa de dispareunia pós-operatória em 1 caso, e recidiva em $25 \%$ dos casos, os sintomas prévios à correção foram, no mínimo, amenizados. Estudos demonstram grau de sa- tisfação adequado com a técnica transperineal, de cerca de $90 \%$ de satisfação ${ }^{2,3}$. A abordagem transanal foi a que apresentou piores resultados, com satisfação de aproximadamente $78 \%$ dos $\operatorname{casos}^{2,3}$. Especificamente no STARR procedure, Slim et al mostram um grau de satisfação de $85 \%{ }^{15}$. Em estudo analisando a técnica transperineal associada a uso de prótese sintética, o grau de satisfação foi de $35,71 \%$.

\section{CONCLUSÃO}

Houve melhora significativa do padrão evacuatório, uma resposta aceitável no tratamento da dispareunia, com índice de recidiva tolerável e alto grau de satisfação pós-operatória. A comparação com as demais técnicas ainda exige estudos comparativos mais significativos, com amostras mais expressivas. Até o momento, qualquer análise comparativa entre as técnicas cirúrgicas utilizadas pode ser falha.

\footnotetext{
ABSTRACT: Rectocele is a poorly diagnosed pelvic dysfunction, despite its significant prevalence. Its recognition is essential for the treatment of certain cases as refractory constipation. The symptoms are vague and not always associated with prolapse. Clinical treatment is ineffective when used alone. There are several techniques, being the transperineal approach an appropriate option for the correction of prolapse. The target of this data is a group of 12 female patients with rectocele: they experienced significant improvement in the defecation standard, an acceptable response in the treatment of dyspareunia, with acceptable recurrence rate and high degree of satisfaction after surgery. The comparison with other techniques still requires significant further comparative studies with samples more expressive. To date, any comparative analysis between the surgical techniques used may be failure.
}

Key words: Retocele, Surgical Repair.

\section{REFERÊNCIAS}

1. Zbar et al. Rectocele: pathogenesis and surgical management. Int J Colorectal Dis (2003) 18:369-384

2. Fischer et al. Functional results after transvaginal, transperineal and transrectal correction of a symptomatic rectocele. Zentralbl Chir. 2005 Oct;130(5):400-4

3. Fischer et al. Transvaginal, transperineal and transrectal approaches for symptomatic rectocele. Coloproctology 2007;29(5):258-264

4. Kahn et al. Techniques of rectocele repair and their effects on bowel function. Int Urogynecol J (1998) 9:37-47

5. Mercer-Jones et al. Outcome after transperineal mesh repair of rectocele: a case series. Dis Colon Rectum, June 2004;47(6):864-868

6. Abendstein et al. The surgical anatomy of rectocele and anterior rectal wall intussusception. Int Urogynecol J (2008) 19:705-710
7. Geoffrey et al. Evaluation and treatment of women with rectocele: focus on associated defecatory and sexual dysfunction. Obstetrics and Ginecology 2004;104(6):14031421

8. Boccasanta et al. Which surgical approach for rectocele? A multicentric report from Italian coloproctologists. Tech Coloproctol (2001) 5:149-156

9. Ommer et al. Results of transperineal levatorplasty in the treatment of symptomatic rectocele. Chirurg (1998) 69: $966 \pm 972$

10. Swift SE, Pound T, Dias JK. Case-control study of etiologic factors in the development of severe pelvic organ prolapse. Int Urogynecol J Pelvic Floor Dysfunct 2001;12:187-92.

11. Samuelsson EC, Arne Victor FT, Tibblin G, Svardsudd KF. Signs of genital prolapse in a Swedish population of women 20 to 59 years of age and possible related factors. Am J Obstet Gynecol 1999;180:299-305. 
12. Richardson AC. The rectovaginal septum revisited: its relationship to rectocele and its importance in rectocele repair. Clin Obstet Gynecol 1993;36:976-83.

13. Watson SJ, Loder PB, Halligan S, et al. Transperineal repair of symptomatic rectocele with Marlex mesh: a clinical, physiological and radiological assessment of treatment. J Am Coll Surg 1996;183:257-61.

14. Sehapayak S. Transrectal repair of rectocele: an extended armamentarium of colorectal surgeons. A report of 355 cases. Dis Colon Rectum 1985;28:422-33.
15. Slim K et al. Repair of rectocele using the Stapled TransAnal Rectal Resection (STARR) technique: intermediate results from a multicenter French study. J Chirurgie 2008; 145(1):27-31.

\section{Endereço para correspondência:}

SINARA MÔNICA DE OLIVEIRA LEITE

Av. do Contorno 4747/912, Serra

CEP. 30110-090

E-mail: sinara.monica@uol.com.br

Contato: 3225-1437 